\title{
Culture des sens, savoirs et transmission du savoir
}

\section{Alain Bouillet}

\section{OpenEdition}

Journals

Édition électronique

URL : http://journals.openedition.org/trema/1916

DOI : 10.4000/trema.1916

ISSN : 2107-0997

\section{Éditeur}

Faculté d'Éducation de l'université de Montpellier

\section{Édition imprimée}

Date de publication : 1 avril 1997

Pagination : 19-32

ISSN : 1167-315X

\section{Référence électronique}

Alain Bouillet, «Culture des sens, savoirs et transmission du savoir », Tréma [En ligne], 11 | 1997, mis en ligne le 01 avril 1997, consulté le 19 avril 2019. URL : http://journals.openedition.org/trema/1916 ; DOI : 10.4000/trema.1916

Ce document a été généré automatiquement le 19 avril 2019

Trema 


\title{
Culture des sens, savoirs et transmission du savoir
}

\author{
Alain Bouillet
}

Interroger les rapports - plus latents que manifestes, plus ancrés dans la pratique que soumis à une tentative d'explicitation, voire de théorisation- que la sensibilité entretient avec le savoir, c'est, d'emblée, énoncer deux questions :

- Quels sont les rapports entre la sensibilité (déclinée suivant les différents « sens » : la vue, l'ouïe, le goût, etc.) et le savoir ? De quel ordre sont les savoirs sensibles ? Quelle place leur est reconnue dans la hiérarchie des savoirs « savants »? Quel statut leur est fait ? En quoi, comment et depuis quand sont-ils pris en compte dans les dispositifs éducatifs et formatifs? Cette question ne peut évidemment prendre sens que si, auparavant on a pris soin de se demander :

- Qu'est ce qu'un savoir sensible ? Quelle est sa spécificité (au regard d'autres savoirs par exemple...) ? Qu'en est-il de sa genèse ? (qui, personne ou institution, le produit, l'établit, le nomme tel, le fait savoir et reconnaître, le rend désirable ? ${ }^{1} \mathrm{Qu}$ 'en est-il de sa transmission : qui transmet quoi, à qui et comment, dans quelle situation, à l'occasion de quel dispositif ? Qu'en est-il, enfin, de son acquisition, de sa compréhension (au sens de prendre avec soi) : le regard s'éduque, mais qui évalue? Qu'est-ce-que savoir regarder? Quand sait-on qu'on le sait?

2 On sent bien (ou plutôt, on subodore; verbe qui dit tout à la fois le pressentiment, l'anticipation, l'intuition d'une vérité cachée et la mise en œuvre d'une sagacité affûtée sachant ne pas s'en tenir à la seule odeur prégnante) que ces deux questions en recèlent bien d'autres (ne seraient-ce que celles nécessaires à les expliciter dans les deux paragraphes précédents). Qu'au fur et à mesure de la réflexion, de la rumination, celles-ci vont s'épanouir comme la fragrance d'un parfum ou le bouquet d'un vin lorsque la main, imprimant au verre un léger mouvement de rotation, permet d'en exhaler l'intensité et la complexité. 


\section{Pourquoi cet intérêt pour les savoirs sensoriels ?}

3 Et peut-être, avant tout autre chose, se demander quel est le sens de cette résurgence de la thématique des sens, du sensoriel et de la sensibilité en cette fin de vingtième siècle, tout particulièrement lorsqu'il s'agit de préoccupations d'ordre éducatif? Pourquoi depuis quinze ans voit-on se multiplier des articles, des ouvrages ${ }^{2}$, des colloques et rencontres diverses (par exemple ceux organisés depuis 1991 à Montpellier sur la vue, le toucher, l'odorat, ...), de multiples expériences pédagogiques (de «La maison des cinq sens " à Paris en 1989 aux classes ayant accueilli depuis trois ans un dispositif « d'éducation au goût », tel que J. Puisais l'expérimente depuis 1974)?

Certes, la prise en compte des sens et du sensoriel (mais quid de la sensibilité ?) dans les préoccupations éducatives n'est pas à vrai dire une innovation. Du «Nous sommes merveilleusement corporel » de Montaigne aux impératifs de «l'éveil sensoriel» des années 1960, en passant par Maria Montessori, l'idée d'une articulation entre le corps et l'esprit, les sens et la raison a de tout temps, sous des formes et avec des fortunes diverses, été une préoccupation des pédagogues. Mais ce qui questionne c'est le soudain intérêt qui semble se développer, tant chez les chercheurs des domaines public (l'Université) ou privé (les laboratoires de l'alimentation industrielle ou des cosmétiques) que dans les écoles maternelles ou primaires, pour ce que l'on appellera ici : la culture des sens.

D'autant plus que cet « engouement» (devrait-on dire : cette mode ?) fait suite à d'autres «irruptions » du même ordre, par exemple le «corps», dans les années 1970. Quelles interprétations donner à un tel phénomène ? Que dire de ces "retours à la nature ", de ces «redécouvertes du corps», de cette "reviviscence du sensoriel»? Réapparition conjoncturelle (comme ces "retours" des «sixties", puis des "seventies", etc. programmés par les «industries de l'imaginaire » et qui rythment sur un mode qui va s'accélérant, la structure du Top 50 : il faudrait alors en faire l'histoire) ou nécessaire régression (comme pour tout processus réellement formatif : il faudrait alors en faire l'analyse au sens psychanalytique du terme); ou bien encore tentative (illusoire) de revenir à l'utopie harmonieuse d'un paradis perdu, où n'existeraient ni la séparation (entre les sexes: le mythe de l'androgyne; entre le sensoriel qui connote les processus primaires et le raisonnable qui témoigne de la secondarisation des pulsions), ni le conflit. Et où, enfin, fusionneraient Nature indomptable et Nature domestique, Intelligence naturelle et Intelligence artificielle. Simple angoisse millénariste (qui ne concernerait alors qu'une partie de l'Occident) ou éternel retour (dont il faudrait questionner la périodicité et l'universalité) ?

Quoi qu'il en soit, le discours sur la culture des sens s'emballe. Anthropologues, économistes, historiens, sociologues et même psychanalystes..., chacun tente de s'emparer de ce nouveau pays de Cocagne. Et le goût, sous les auspices de l'art culinaire, n'est pas le dernier des sens à générer une telle pratique. A tel point que N. StrouzePlessis et $\mathrm{H}$. Strohl ${ }^{4}$ se demandent si « ce phénomène ne serait pas à la cuisine, ce que la récession ethnologique est à bien des cultures étudiées, la mise en paroles frénétique d'une culture déjà morte " ou la recherche nostalgique d'un paradis culinaire perdu. Serions-nous en train, vieux réflexe cannibale, d'incorporer métaphoriquement, une culture traditionnelle qui meurt sous nos yeux? Dernière tentative de nous approprier sa sagesse? 


\section{... Même en Sciences de l'Education}

7 A leur tour, praticiens et chercheurs des Sciences de l'Education semblent, encore bien modestement, vouloir s'intéresser à ce domaine : au-delà des effets d'entraînement que créent les programmes de recherche, on peut recenser plusieurs raisons.

1. Les Sciences de l'Education ne peuvent ignorer les débats "philosophiques qui, de Parmenide à Michel Serres ont animé la question de savoir en quoi et jusqu'à quel point les sens contribuaient à la connaissance (et à la reconnaissance) du monde; le plus souvent pour en signaler les limites, les aberrations et les faiblesses.

2. Les activités sensorielles (leur éveil, leur développement, leur orientation) ont fait l'objet de nombreuses réflexions de la part des théoriciens de l'éducation et plusieurs dispositifs pédagogiques ont tenté de donner à celles-ci un début de réalisation concrète. En outre, l'éducation artistique (de la méthode Guillaume en 1880 à «l'Hermès éducateur » de Bruno Duborgel en $1990^{5}$ demeure une préoccupation constante de l'école maternelle et de l'école élémentaire, du moins pour ce qui concerne les arts plastiques et, dans une moindre mesure, la musique. Quant à l'odorat, au toucher, au goût, parents pauvres de l'esthétique, ils ne sont pour l'instant que l'objet de tentatives expérientielles très isolées.

3. Cependant, l'éducation, ce n'est pas seulement le dispositif institué de la scolarité. Et, même après avoir interrogé l'école maternelle, primaire et secondaire, sans oublier l'enseignement technique qui est sans doute l'un de ceux où une culture des sens est la plus mise à l'épreuve sans pour autant être, d'ailleurs, théorisée (que l'on pense à l'apprentissage des métiers du bois, des métaux, de la pierre, aux écoles hôtelières, à l'ensemble des cultures techniques auxquelles sera fait référence plus loin), il reste à explorer le domaine de l'éducation non formelle. Ainsi nomme t-on celle qui, implicitement, s'opère à l'intérieur de l'espace et du groupe familial, entre pairs dans la rue ou aux champs, dans l'espace multidimensionnel des relations qui se nouent avec les autres, sous le regard omniprésent des médias audio-visuels, etc. Processus incessant, souterrain, rhizomique, d'autant plus difficilement repérable pour un observateur extérieur que la plus grande part en demeure étrangère à la conscience du sujet et que celle qui affleure ne se dit que de façon laconique, lacunaire et avec de multiples réticences. C'est d'ailleurs l'une des spécificités de ces savoirs sensibles que de se prêter malaisément, voire de se soustraire ou se dérober, à la verbalisation. Que l'on pense ici aux pratiques éducatives qui souvent achoppent, en formation initiale comme en formation continuée, sur les difficultés qu'ont les enseignés ou les formés à exprimer leurs sentiments ou leurs émotions face à une œuvre picturale (et bien plus encore quand il s'agit de rendre compte de ce que l'on a entendu, dans le cas d'une écoute collective des sons, et comment...). On retrouverait ces mêmes difficultés lors des tentatives de constitution de «systèmes experts » à partir des cultures techniques professionnelles.

8 Réticences à formuler des savoirs par essence ineffables ou résistance à livrer l'intimité de ses émotions ou à se laisser dépouiller de ses savoirs expérientiels ? Là encore, il faudrait questionner plus avant. L'éducation informelle apparaît donc comme ce lieu d'élection où prennent forme, s'élaborent, se développent, se mettent à l'épreuve, se confortent les cultures des sens spécifiques à l'auto et à la socio-poièse de chacun.

\section{Du goût et du savoir...}

9 A ces trois raisons principales, (il y en aurait sans doute bien d'autres, adjacentes, dérivées, subreptices...), il faut encore en ajouter une quatrième, qui tient aux rapports étymologiques qu'entretiennent les substantifs nommant un sens (par exemple: 
l'odorat), leurs organes (le nez), les adjectifs et adjectifs substantivés qui pointent les qualités de l'organe ou de son utilisateur (sagace, sagacité), et quelques termes privilégiés du vocabulaire de l'éducation; ne serait ce par exemple que le couple « nourrir-éduquer » qui offre, à qui veut bien prendre le temps de le déployer, de multiples pistes de réflexion.

Ainsi en va-t-il pour le " goût », du moins dans nos cultures latines (on verra plus loin ce qu'il en est des rapports que les anciens Grecs tissaient entre la vue et le savoir). « L'oubli vient un peu trop vite ", écrit M. Serres ${ }^{6}$, « de ce que l'homo sapiens désigne qui réagit à la sapidité, qu'il l'apprécie et la recherche, à qui le sens du goût importe, bête à saveur, avant de vouloir dire l'homme devenu tel par jugement, intelligence ou sagesse, avant de dire l'homme parlant [...]. La sagesse vient après le goût, elle ne peut advenir sans lui, mais l'oublie. » Michel Serres le remarque, Georges Haldas ${ }^{7}$ le confirme : Platon dans le Banquet l'avait bien vu, et les Pères de l'Eglise, et Dante, bien d'autres qui ont noté que saveur (des mets) et savoir (sapience) ont une étymologie commune : le latin «sapere ». Et Martine Chatelain-Courtois théorise: "On oublie trop que savoir, étymologiquement signifie 'goûter' (latin : sapere), et que c'est un mot parent de saveur. Il faut l'argot pour nous rappeler les sources sensorielles de la connaissance, avec l'expression « être au parfum $»^{8}$.

11 Le rapport entre langage et sensibilité est très largement métaphorique, ce qui, peut-être, témoigne à nouveau des résistances que la sensibilité oppose à se laisser verbaliser, de l'ensemble des raisons qui font que les informations venues des sens empruntent de façon privilégiée d'autres modes d'expression que le discours analytico-descriptif: la métaphore, l'allégorie, le discours poétique, voire l'expression non verbale. Ce qui, entre autres, révèle un problème méthodologique: comment explorer le sensible dans ses rapports avec le savoir? Dans quel métalangage, qui ne froisse ni « la bouche d'or » celle qui discourt, ni la "goûteuse ", celle qui savoure, dire ce lien, sans l'affadir ou le réduire dans l'alchimie de l'analyse?

On conçoit peut-être mieux à présent ce qui peut intéresser théoriciens et praticiens de l'éducation dans l'étude d'une telle culture des sens :

1. la reconnaissance d'un champ du savoir qui, pour l'instant, leur échappe encore en grande partie. Mais peut-être faut-il également entendre reconnaissance dans le sens de possibilité d'être, pour la culture des sens, reconnue comme « savoir » et donc, de prétendre à l'intérêt des pédagogues ;

2. la compréhension de ce qui œuvre dans les «savoirs sensibles" pourrait peut-être permettre de penser leur articulation avec les «savoirs scolaires" et, de ce fait, l'articulation entre éducation formelle et éducation informelle ;

3. savoir comment s'élabore, s'acquiert et se transmet la «culture des sens " permettrait d'en envisager l'éducation et la possibilité d'intervenir ainsi soit dans le sens de la conservation de ces savoirs (un bon nombre d'entre eux sont menacés de disparition), soit contre les processus qui tendent à travailler à leur disparition (par exemple, les efforts actuellement déployés pour maintenir une culture du goût).

13 Deux exemples vont permettre de reprendre et de prolonger les réflexions précédentes. Le premier, partant de la constatation que le voir est construit, que l'on ne voit que ce que l'on connait, tente de comprendre comment, au cours des siècles, nous avons « appris à voir ", et en quoi, socialement produit, le regard est en retour générateur de savoir. Le second interroge la genèse, la pratique et la transmission d'une culture des sens dans ses activités techniques qui lient l'homme à l'animal d'élevage et aux plantes cultivées et questionne l'articulation de celle-ci avec les savoirs « savants ». 


\section{Le « voir » est construit}

14 Parmi les sens qui médiatisent, filtrent et organisent notre relation au monde, la vue (et corrélativement : le regard) a une importance toute particulière. Sens de la «distance » (comme l'ouie) $)^{9}$, valorisé chez l'être humain à l'occasion de son "redressement $»^{10}$, privilégié en Occident, et plus précisément en Italie à l'aube du XVe siècle, comme voie principale par laquelle l'intellect se saisit de son environnement ${ }^{11}$, générateur de fantasmes d'emprise totalitaire en ce vingtième siècle finissant ${ }^{12}, c^{\prime}$ est par lui que le réel " nous saute aux yeux », acquérant ainsi ses caractéristiques de vérité et d'évidence. Or, rien de moins évident que cette " évidence » là. Même si, pour les savants grecs que nous considérons, à présent, comme des "philosophes " en déniant à leur théorie un statut scientifique), la vue fut sans contexte le plus important des cinq sens Aristote la désigne comme le meilleur des sens; Platon dit que la vue est la plus aiguë des perceptions sensorielles, que l'œil est considéré comme la plus belle partie du corps humain et que la pupille - en grec la koré, la jeune fille et en latin, pupilla, la poupée - est la meilleure partie de l'œil) ${ }^{13}$, il demeure que, face à la " vérité » de ce que nous percevons, ils n'ont cessé, de Platon jusqu'aux dernières recherches des cogniticiens contemporains, de nous mettre en garde quant à la « tromperie des apparences ».

Nous ne reviendrons pas ici sur cette question complexe pour savoir en quoi, comment, et jusqu'où nos sens, plus spécifiquement la vue, nous abusent, ce qui nous amènerait, entre autres à devoir distinguer entre savoirs stables et savoirs instables ${ }^{14}$, mais ce qu'il faut retenir de ce débat séculaire, c'est que le voir est construit, et que toute perception visuelle est une procédure d'une extrême complexité à l'intérieur de laquelle l'œil et l'esprit $^{15}$, sensation et intellect, dispositif physiologique et processus psychologique, interférent, se combinent, s'articulent et s'enchevêtrent étroitement. «L'œil innocent ne voit rien » affirme E.H. Gombrich ${ }^{16}$ et, ajoute Pierre Francastel : «On ne voit que ce qu'on connaît, ou du moins ce qu'on peut intégrer dans un système cohérent et par suite déployé dans le temps des représentations significatives $» \cdot{ }^{17} \mathrm{En}$ fait, il n'y aurait pas de perception visuelle sans mise en œuvre de multiples processus cognitifs : «choix » d'un champ dans lequel exercer la vision (choix, entre guillemets, car celui-ci est fonction de multiples facteurs: les capacités ophtalmologiques de l'œil humain, avant tout, mais également la nature de l'objet, et les rapports qu'entretient la personne avec celui-ci); choix à l'intérieur de ce champ d'une zone restreinte, permettant à la vision de s'effectuer avec netteté (limitation et précision des informations dans un laps de temps déterminé). A ces activités de sélection et de combinaison des informations, il faut ajouter la capacité d'abstraire l'objet perçu du contexte dans lequel on le perçoit, ce qui permet d'identifier (de reconnaître) les objets quelle que soit la distance à laquelle on les observe, ce qui suppose l'existence d'une pensée logique au niveau même de la perception.

Ces caractéristiques de la "pensée visuelle $»^{18}$ nous incitent à penser que vision et connaissance ont partie liée et qu'ainsi que le mentionne E.H. Gombrich : nous ne voyons que ce que nous remarquons, ne remarquons que ce que nous cherchons, ne cherchons que « [...] lorsqu'une disparité quelconque éveille notre attention, lorsqu'un désaccord se produit entre ce que nous attendions et le message qui nous parvient. $»^{19}$ 


\section{Le « voir » : socialement produit et générateur de savoir}

17 L'interrogation sociologique vient ici relayer les travaux des psychologues et des cogniticiens. Dans un ouvrage novateur, de ceux qui "donnent à penser», Anne Sauvageot ${ }^{20}$ défend la thèse suivant laquelle le regard serait socialement produit en ce sens «[...] qu'il est conditionné par le jeu des formes que lui offre tout un environnement ».

"Les figures et les images qui nous entourent, même les plus banales", précise t-elle, " concourent à édifier des images-normes instaurant chez les individus un habitus tout à la fois perceptif, cognitif et symbolique. L'apprentissage normatif de la perception se double en effet d'une structuration symbolique qu'active l'intégration de modèles référents. Car les formes qui constituent notre environnement visuel ne sont pas innocentes en ce qu'elles expriment un certain état d'être culturel de la matière. Et à ce titre, l'organisation du visible constitue une sorte de 'grammaire sémantique' qu'intègre le regard à son contact. Une phénoménologie de la matière - la façon dont celle-ci est façonnée par des rationalités et vécue par la conscience - peut donc instruire tant sur l'évolution de nos conduites perceptives que sur le rôle de la vision dans la dynamique de la connaissance ».

Mais l'intérêt de la thèse d'Anne Sauvageot tient dans le fait que si le regard est socialement produit, il est également producteur de social, puisqu'en retour le voir est générateur de savoir. "La construction du savoir », poursuit-elle, " emprunte en effet à l'organisation du voir ses principes structurants de telle sorte que l'évolution des schémas perceptifs entraîne avec elle celle des rationalités. » Selon R. Arnheim, ${ }^{21}$ " [...] les formes sont des forces formantes puisque l'élaboration des concepts commence avec la perception des formes. » La matière, les savoirs et le voir sont donc indissociables dans leurs modélisations réciproques et la mise en lumière de leurs interactions contribue à ce qu'Anne Sauvageot appelle une sociologie du regard. Celle-ci aurait alors pour tâche « de saisir la façon dont un ordre visuel participe d'une construction sociale de la réalité. »

"Apprendre à voir " n'est donc pas seulement une formule de rhétorique et l'on peut penser que ce qui vaut pour le développement de la personne vaudrait également, à l'échelle des sociétés. Les documents manquent qui permettraient de suivre les modifications de la vision à travers les siècles, mais l'évolution des modes figuratifs permet d'entrevoir les transformations du regard. En effet, chacun des grands moments culturels, dans toutes les civilisations, met en œuvre de nouvelles conventions figuratives (c'est ce qu'il faut entendre par « ordre visuel »), dont on peut penser qu'elles ne sont pas seulement l'expression de systèmes symboliques, mais l'actualisation de modes visuels novateurs. Ces "grands moments", Anne Sauvageot en repère trois qui, chacun, renvoient à des épisodes importants de l'évolution des connaissances, organisant pour chacun d'entre eux une matérialité qui correspond aux différents « styles du connaître ». Chacune de ces époques assure le primat d'une certaine logique de la nature qui soustend une spéculation scientifique et philosophique de la matière, en même temps qu'une relation concrète avec elle par l'intermédiaire des arts et des techniques.

Pour le monde grec, et en particulier pour Platon, le Cosmos est le modèle, idéal et idéel, du monde matériel. Les temples comme les statues de l'âge classique, obéissant à la mimésis qui se doit de traduire cet idéal cosmique d'ordre et d'harmonie, peuvent être compris comme les paradigmes de la vision que les Grecs portaient sur le monde, exemplaires de la mesure qu'ils exerçaient sur lui. Avec la Renaissance, d'un espace tactile (« toucher des yeux » on passe à un espace dominé par l'optique, à l'image mécanique d'un 
monde qui s'annonce, monde homogène comme l'attestent les nouvelles règles figuratives de l'espace. La perspective immobilise les objets, inscrivant les hommes dans un rapport de distanciation et d'objectivation au monde qui pèsera sur les modes de représentation jusqu'à la fin du XIXe siècle. Avec la Modernité (et la post-modernité...), le monde matériel renouerait avec le désordre. Un espace numérique succède à l'ancien espace optique, espace saturé de stimuli visuels, privilégiant les processus et «[...] faisant basculer le monde de la physis, comme celui du social dans l'instabilité, l'aléatoire, l'incertitude, la simulation [...] $\aleph^{22}$ L'émergence de nouveaux espaces perceptifs implique donc de nouvelles appréhensions du monde. Ce que l'œil déchiffre au contact de son environnement alimente une expérience perceptive qui, au gré des codes visuels qu'elle favorise devient, progressivement usualisation du monde. C'est en ce sens que Paul Virilio ${ }^{23}$ peut parler de " générations visuelles ».

Hormis la vue (et l'histoire du regard), à côté de l'esthétique (entendue comme compétence à développer sa sensibilité dans les domaines "artistiques »), la culture des sens trouve à s'exercer de multiples façons: connaître et reconnaître au toucher l'essence, la qualité et le fil d'un bois; saisir à l'œil, ainsi qu'au tact en ressentant la résistance qu'il oppose via la truelle à la main qui le «touille », le degré de fluidité ou de compacité d'un mortier et savoir s'il faut rajouter du sable (de quelle nature, de quelle granulométrie, de quelle couleur ?), de la chaux, du ciment (en quelles proportions ?), de l'eau (en quelle quantité ?), en fonction de quelle utilisation (maçonnerie de pierres dures, de briques, de tuiles, de parpaings ou mortier à joints, à enduit, etc.) ; savoir quand arrêter la réduction d'une sauce, la prise d'un caramel, la cuisson des pâtes etc., met en œuvre de multiples savoirs sensoriels : l'odorat, la vue, le toucher, le goût pour les sauces, le caramel et les pâtes ; l'ouïe pour le grésillement de l'andouillette sur la braise, la vue, le toucher (via le fil du couteau ou le manche de la fourchette à gigot) pour la cuisson des viandes.

\section{Le « coup d'œil » de l'éleveur}

Le choix d'exemples empruntés au monde rural, et plus particulièrement au travail, aux métiers agricoles, ainsi qu'aux mécanismes de l'acquisition et de la transmission des savoirs à travers le groupe familial et territorial des agriculteurs, tient au fait que là, peut-être plus qu'ailleurs, malgré «le terrorisme scientifique et technique des agronomes, des décideurs et des vulgarisateurs $»^{24}$, a pu se maintenir, jusqu'à une date récente, une culture des sens liée aux relations complexes que l'homme, éleveur ou maraîcher, entretient avec l'animal d'élevage ou les plantes cultivées, c'est-à-dire avec le vivant. Cette culture des sens sous-tend en permanence l'activité technique des paysans, laquelle demeure étrangement ignorée des institutions de formation, de vulgarisation et de gestion agronomiques. Il y a à cela de multiples raisons, politiques, économiques, mais également des raisons qui tiennent à la spécificité même des activités et que François Sigault expose en ces termes :

"A la différence du raisonnement scientifique qui est général, explicite et désintéressé, le raisonnement technique est singulier (il dépend de circonstances particulières), implicite (la plus grande partie de ses éléments sont inexprimés) et motivé (l'action technique est toujours immédiatement utile). C'est pourquoi ses structures n'apparaissent pas à l'observation superficielle. $»^{25}$ 

tout jeune enfant. $\aleph^{27}$ Cette phrase de La voie peule, recueil sur les apprentissages, les pratiques et les connaissances des pasteurs Peuls, dit bien la nature du lien qui se tisse entre l'éleveur et l'animal et qu'à l'occasion d'autres élevages, celui des humains, on nomme : l'attachement. ${ }^{28} \mathrm{~L}$ 'attachement se développe dès la naissance des bêtes lorsque les éleveurs prennent soin des agneaux ou des veaux. La qualité de celui-ci va ensuite déterminer la plus ou moins grande facilité que l'éleveur aura pour travailler avec l'animal, pour l'approcher, le traiter et le soigner. Cela se traduira également par l'apprentissage du côté de la bête d'un certain nombre de conduites (approcher quand on les appelle, accepter qu'on les panse sans résistance) et donc se fera au moindre coût d'énergie physique et psychique. Quand l'attachement est réalisé, l'animal se sent protégé et pris en charge par l'éleveur, par le corps tout entier, la voix, les attouchements : c'est d'un véritable "holding " au sens de $\mathrm{D}$. Winnicott ${ }^{29}$ dont il s'agit. Ce phénomène va se développer à partir des liens qui se tissent avec l'éleveur, à travers l'expression de son corps (présence), de sa gestuelle, de sa voix, voire de son odeur. bonne mère et se montrer attentif aux besoins de ses bêtes. Cependant, D.W. Winnicott le 
signale, « les bonnes mères qui prévoient et précèdent tous les besoins du nourrisson, ne lui donnent pas la possibilité de ressentir de la frustration ni d'exprimer des demandes et d'expérimenter une autonomie progressive $» .{ }^{30}$ Pour les bêtes, il en irait de même et certains éleveurs surmaternent leur troupeau.

\section{Le métier et la transmission des savoirs} été quasiment ignorée, à l'exception des maisons familiales rurales qui ont perçu son importance) que se réalise la majeure partie de ces apprentissages. Les enfants accèdent aux apprentissages de pratiques et de découpages conceptuels en se nourrissant de toute l'ambiance dans laquelle ils baignent dès le plus jeune âge et où les discussions entre adultes jouent un rôle pédagogique aussi important que l'apprentissage technique proprement dit. L'enfant est accueilli dans un bain de paroles dès la naissance, qui va véhiculer des taxinomies, une manière de définir, à partir de la pratique, le monde des objets techniques, des gestes, des postures, des relations avec le monde, les autres, l'espace et le temps. C'est dans cette parole qui ne le quitte pas que l'enfant va construire, catégoriser l'univers technique et social qui l'entoure. Ce processus de «nourrissage » de l'enfant n'est pas réglé comme dans l'apprentissage initiatique par des rituels stricts incluant des étapes d'isolement, de souffrance, prodiguées par le groupe sur le futur initié, ni par des maîtres connus d'avance. C'est un processus graduel. La seule grande rupture dans l'apprentissage du métier d'éleveur ovin est la garde en montagne qui est un sevrage (chez les éleveurs pyrénéens on dit : "despopar» : enlever un enfant au sein de sa mère), un "désencordage " de la mère et de la maison, un rite de passage. Cette « empreinte » des savoirs et savoir-faire avec le vivant, végétal et animal, va se former à travers une activité de jeu : l'enfant se familiarise, tout petit, dès qu'il se débrouille tout seul, avec l'intimité des bêtes et du jardin, de la terre et de l'eau. Puis le jeu deviendra travail, prise en charge, responsabilisation vers quatorze, quinze ans. " Moi, j'ai un fils, à huit ans, il a pris toutes les brebis de Sentenac et il les a amenées ici, à Casabède, à huit ans ! Mais il peut y venir, il est capable de... Seulement, il m'a vu faire, je l'y ai fait tester, il est capable de faire quelque chose... ${ }^{31}$

C'est donc dans la culture sensible, dans la parole qui dit la pensée technique du groupe que l'enfant va puiser les taxinomies indispensables. Ces systèmes de nomination très sophistiqués et opératoires qui collent à la diversité du territoire local et à sa spécificité n'ont pas d'équivalents dans les classifications scientifiques. En outre les mots seront mieux assimilés selon la relation que l'on entretient avec celui qui les prononce. L'enfant va acquérir également plus facilement les expressions, les images, les métaphores qui guident l'action technique et le matériel symbolique véhiculé par la personne à laquelle il s'identifie. 

d'identification telles que l'on peut les rencontrer dans les dispositifs éducatifs. En effet ces apprentissages sont marqués par l'intensité et la qualité des relations au père. Les garçons apprendront ou rejetteront avec d'autant plus de facilité certains gestes et certains savoirs concernant telle ou telle production en fonction de la tonalité de cette relation au père. Dans le cas d'un rejet des pratiques et productions paternelles, ils revivent adultes cette contre-identification avec beaucoup d'émotion et de violence lorsqu'ils analysent dans leurs choix d'orientation les facteurs qui ont déterminés ceuxci : «j'ai fait du mouton, car cela ne plaisait pas au père, il n'aimait que la vache, il ne voulait pas, ça été dur.» De nombreux éleveurs d'élite insistent sur le rôle joué par un oncle, un grand-père lui-même producteur de bêtes et pour qui ils avaient un grand attachement. Certains évoquent même une parenté fantasmatique. ${ }^{32}$

Peut-être est-on mieux à même de comprendre à présent pourquoi cette culture des sens est si difficile à repérer, à identifier, à comprendre et que ce n'est, comme le rappelle Michèle Salmona, que «dans la dynamique de recherche-action menée sur une longue durée, et dans une relation de compagnonnage et d'échanges réciproques avec les paysans de micro-territoires que (l'on peut) tenter de déchiffrer la complexité cognitive, affective, symbolique, imaginaire et actuelle de leurs métiers et cultures techniques. " $^{33}$

Gravure d'époque. Début du XXe $\mathbf{s}$.

Ch. 77, p. 60 :La louée. - Un petit berger, extr. deLes Bucoliques, de J. RENARD.

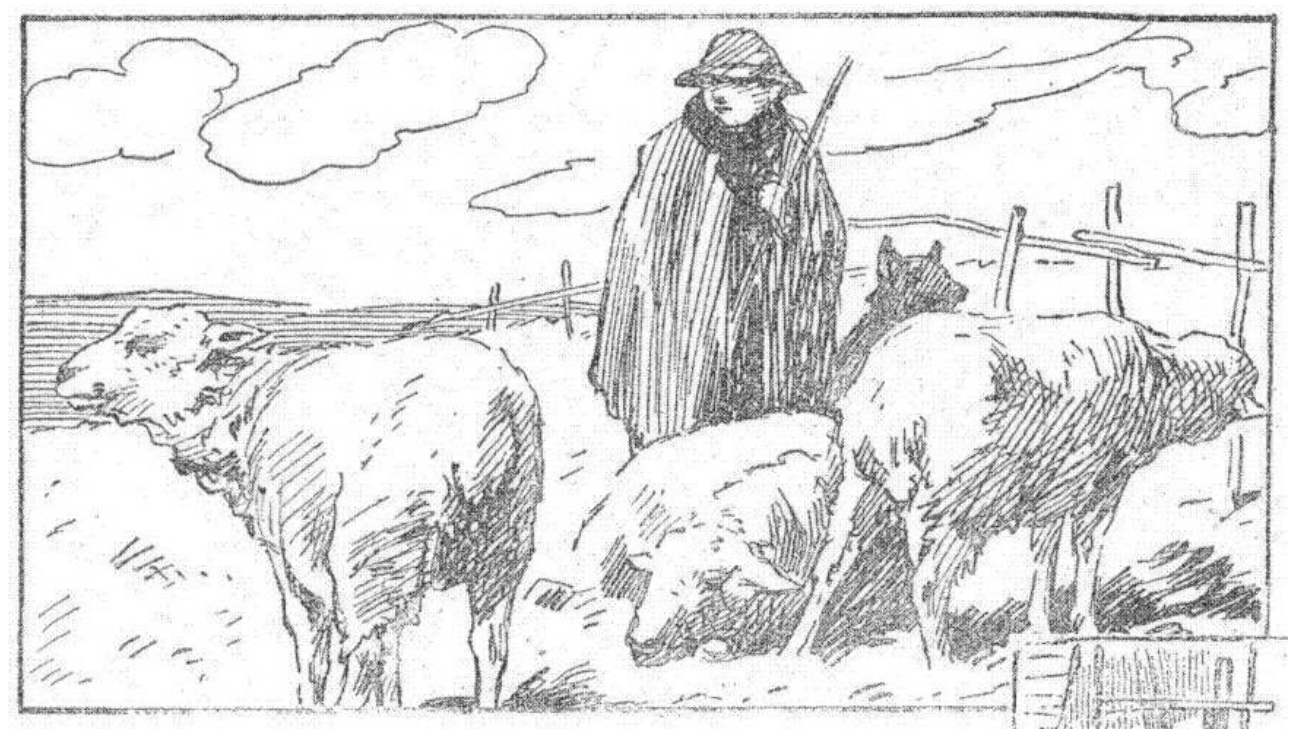

CHADEYRAS F. (dir. d'Éc. Normale), Belles Lectures Françaises, (C. moyen \& sup.), Paris, Delagrave, 1924. 
Gravure d'époque. Fin du XIXes.

Ch. : Les Paysans et les Ouvriers, p. 18.

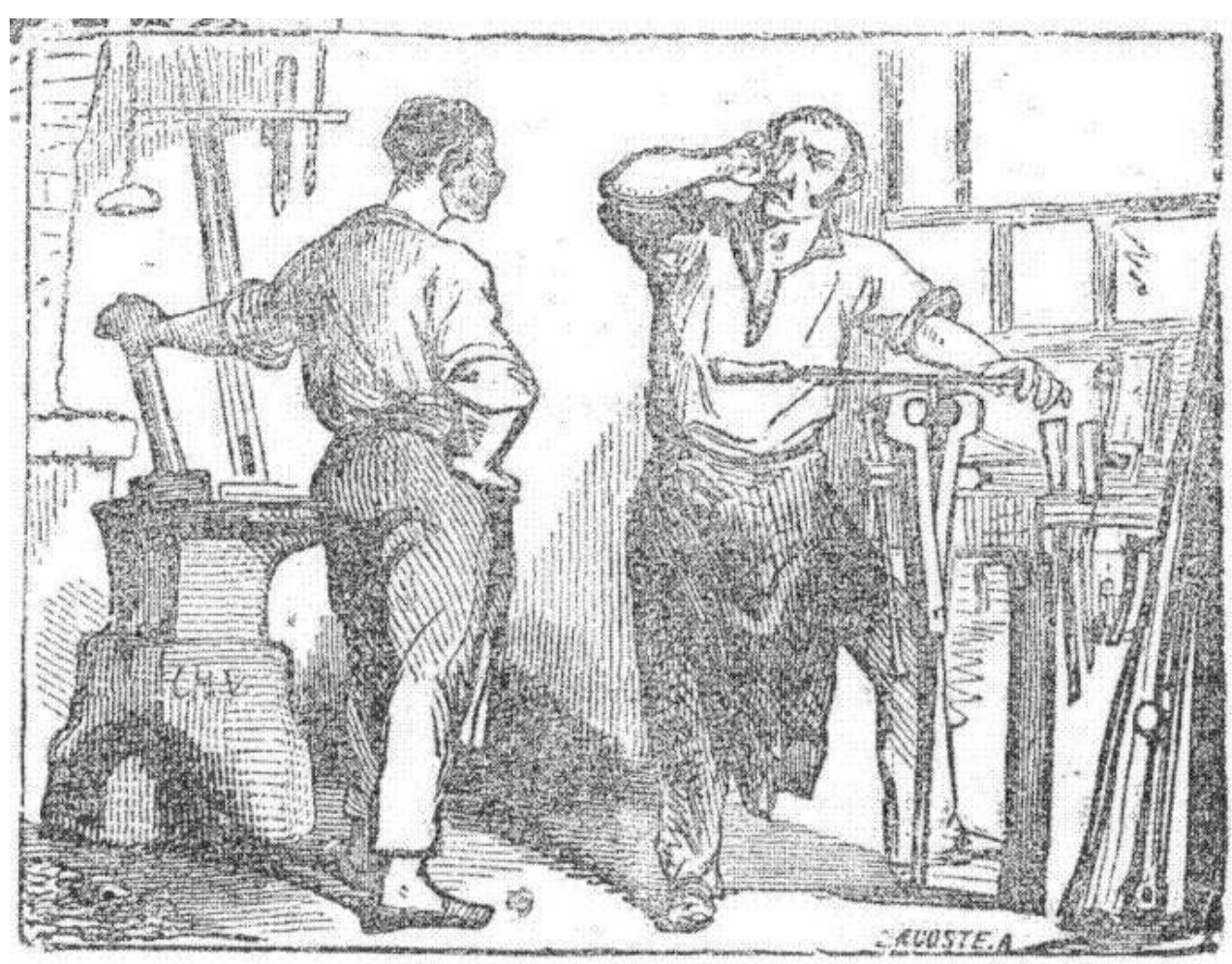

HENRY F., Les Chants de l'Enfance - Rondes et Chansonnettes Enfantines, sur des airs connus, (Livre I, Rondes et Chansonnettes pour petits garçons), Paris, Éd./Typographe : Ch. Noblet, 1881.

\section{NOTES}

1. C'est en l'occurrence, une question qui, - actuellement -.travaille la pratique des « réseaux d'échange de savoir ». cf.: HEBER-SUFFRI C. et HEBER-SUFFRI M., Le cercle des savoirs reconnus, Paris, Desclée de Brouwer.

2. CAMPORESI P., L'officine des sens, 1989 ; CHATELET N., Le corps à corps culinaire, 1977 ; HAR-RUS G., La Psychanalyse de la gourmandise, 1994; LE GUERER A., Le pouvoir de l'odeur, 1988; SERRE M., Les cinq sens, 1985. Revues : «Odeurs », in Autrement, $\mathrm{N}^{\circ} 92,1987$. « Nourritures », in Autrement, №108, 1989. «Nourritures d'enfance", in Autrement, №129, 1991. "Les cinq sens", in Science et Vie, №158, mars 1987.

3. $c f$. : FLICHY P., Les industries de l'imaginaire, Grenoble, P.U.G., 2e éd., 275 p., 1991.

4. STROUZE-PLESSIS N.et STROHL H., «La connaissance du mangeur », in: DUVIGNAUD J., Sociologie de la connaissance, Paris, Payot, 1979, p. 238.

5. DUBORGEL B., "Scènes de l'enseignement artistique », in : LAGOUTTE D., Les arts plastiques contenus, enjeux et finalités, Paris, A. Colin, Coll. - Formation des enseignants, 1990, pp. 71-90

6. SERRES M., Les cinq sens, Paris, Grasset, 1985, p. 167 
7. HALDAS G., La légende des repas, Paris/Lausanne, Éd. Juilliard, Coll. - L'âge d'homme, 191 p., 1987.

8. CHATELAIN-COURTOIS M., Les mots du vin et de l'ivresse, Paris, Belin, 303 p., 1984.

9. Les sens peuvent être classés selon la proximité de la source de sensations qu'ils permettent de percevoir. La «vue » et « l'ouïe » seraient des sens du lointain, le goût et le tact impliquent la proximité voire l'introjection, l'odorat serait un sens intermédiaire.

10. $c f$., FREUD S. : La vue contre l'odorat... Ce que dit S. Freud sur la naissance de l'érotique visuelle et les origines de la famille.

11. Tel que le firent Brunelleschi et Alberti, $c f$. Hubert DAMISCH H., in Les origines de la perspective, Paris, Flammarion, 1987.

12. «Du panoptique de Bentham à la video surveillance [...] des images matérielles aux images virtuelles. De la perception de la réalité à la deréalisation », cf. VIRILIO P., in La machine de vision, Paris, Galilée, 1988.

13. SIMON (Gérard), Les regards, l'être et l'apparence dans l'optique de l'Antiquité, Paris, Éd. Du Seuil, 1988.

14. Savoirs stables : ceux qui permettent de repérer des indices stables et de les interpréter. (Ex. : la composition du Coca-Cola). Les savoirs instables mettent en oeuvre des procédures plus complexes à partir de critères variables (Ex. : Savoir reconnaître un château Haut-Brion, 1961).

15. Pour reprendre le titre d'un ouvrage de MERLEAU-PONTY M., L'oeil et l'esprit, Paris, Gallimard, 1964.

16. GOMBRICH E.H., L'Art et l'illusion, Paris, Gallimard, 1987

17. FRANCASTEL P., Etudes de sociologie de l'art, Paris, Denoél/Gonthier, 1976, p. 60.

18. La pensée visuelle est le tire d'un ouvrage de : ARNHEIM R., Paris, Flammarion, 1976.

19. GOMBRICH E.H., op. cit.

20. SAUVAGEOT A., Voirs et Savoirs. Esquisse d'une sociologie du regard, Paris, PUF, Coll. - sociologie d'aujourd'hui, 249 p., 1994.

21. ARNHEIM R., op. cit.

22. SAUVAGEOT A., op. cit

23. VIRILIO P., La machine de vision. Paris, Éd. Galilée, 1988

24. SALMONA M., Les paysans Français: Le travail, les métiers, la transmission des savoirs, Paris, L'Harmattan, 372 p., 1994.

25. SIGAULT F, Ecologie et Sciences Humaines, (texte présenté au Centre de Recherches Comparatives et Interdisciplinaires, groupe international), 1974, (in: SalmonaM., op. cit. p. 2)

26. HAUDRICOURT A.G, L'homme et les plantes cultivées, Paris, A.M. Métaillé, 1987. - cf. aussi, avec DIBIE P., Les pieds sur terre, Paris, A.M. Métaillé, 1987.

27. DIOULDÉ L., La tradition peule des animaux d'attache, Doctorat de $3^{\text {e }}$ cycle en ethnologie. Université de Paris X, Nanterre, 1973.

28. CYRULNIK B., Sous le signe du lien, Paris, Hachette, 1989.

29. WINNICOTT D., De la pédiatrie à la psychanalyse, Paris, Payot, 1969.

30. WINNICOTT D., op. cit.

31. BESCHE-COMMENGE B., Les savoirs des bergers de Casabède, Toulouse, Université de Toulouse Le Mirail, Travaux de l'Institut d'Etudes Méridionales, 1977.

32. cf.: EIGUER A., La parenté fantasmatique. Paris, Dunod, 1987.

33. SALMONA M., op. cit. 


\section{RÉSUMÉS}

Quels sont les rapports entre la sensibilité et le savoir? Qu'est-ce qu'un savoir sensible? Quelle est sa spécificité, sa genèse ? Comment se transmet-il ? Comment se l'approprie-t-on ? Il y a là des questions qui, en cette fin du vingtième siècle, commencent à devenir des préoccupations éducatives.

Pourquoi cet intérêt pour les savoirs sensoriels de la part des professionnels de l'éducation? Qu'en est-il de la transmission informelle d'une culture des sens? Deux exemples prolongent ces interrogations: celui de l'évolution du regard en occident et celui de la genèse, de la pratique et de la transmission d'une culture des sens dans les activités qui lient l'homme à l'animal d'élevage et aux plantes cultivées.

What is the relationship between sensitivity and knowledge? And what is a knowledge based on the senses? What is its specificity, its genesis? How is it transmitted.? How is it appropriated? Now in the last years of this our 20th century, these questions are beginning to point to educational concerns.

Why this sudden interest for the knowledge of the senses on the part of the professionals in the world of education? What may be said of the informal transmission of a culture based on such knowledge? Two examples will illustrate this discussion : first, the evolution of the critical eye in western culture, then the genesis, the practice and the transmission of a culture of the senses in the activities linking man to the domesticated animals and plants.

\section{INDEX}

Mots-clés : culture, esthétique, savoir, sensibilité

Keywords : aesthetic, culture, knowledge, sensitivity

\section{AUTEUR}

\section{ALAIN BOUILLET}

Maître de conférences, Université de Montpellier III 